\title{
Condition Structural Index using Principal Component Analysis for undamaged, damage and repair conditions of carbon fiber-reinforced plastic laminate
}

\begin{abstract}
This article deals with the data reduction technique using the principal component analysis applied to the carbon fiber-reinforced plastic panels for structural health monitoring approaches. Two carbon fiber-reinforced plastic panels subjected to damage and repair coincide with typical aircraft repair procedures found in the aircraft structural repair manual. The panels were simulated with $30 \mathrm{~mm}$ diameter of partial and full penetration damages using a diamond-coated router. The data (50 observations) were captured for the undamaged, damaged, and repaired conditions by placing lead zirconate titanate smart sensors at $100 \mathrm{~mm}$ across the damaged and repaired structures. A time-based data response was captured for post analysis during the interrogation on the structure at each condition. The raw data were captured in a Lamb waveform, and the interested features were extracted using Morlet wavelet analysis to evaluate the Condition Structural Index and Amplitude-Based Assessment for each condition retrieved from the Gaussian-like distribution. The results were evaluated using the principal component analysis technique in order to distinguish the characteristic of the undamaged, damaged, and repaired conditions. The results showed that in all cases considered, it was possible to distinguish the conditions of undamaged, damaged, and repaired states with promising accuracy and repeatability of the data.
\end{abstract}

Keyword: Carbon fiber-reinforced plastic laminates; Principal component analysis; Lead zirconate titanate sensors 